\title{
Heterogeneous and hyperfine interactions between valence states of molecular iodine correlating with the $I\left({ }^{2} \mathbf{P}_{1 / 2}\right)+I\left({ }^{2} \mathbf{P}_{1 / 2}\right)$ dissociation limit
}

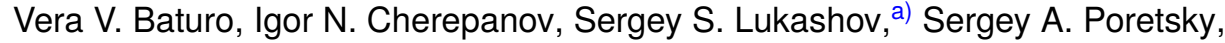 \\ Anatoly M. Pravilov, and Anatoly I. Zhironkin \\ Department of Physics, Saint-Petersburg State University, SPbU, 7/9 Universitetskaya Nab., \\ St. Petersburg 199034, Russia
}

(Received 29 December 2015; accepted 22 April 2016; published online 13 May 2016)

\begin{abstract}
Detailed analysis of interactions between all $0_{g}^{+}, 1_{u}$, and $0_{u}^{-}$weakly bound states of iodine molecule correlating with the $\mathrm{I}\left({ }^{2} \mathrm{P}_{1 / 2}\right)+\mathrm{I}\left({ }^{2} \mathrm{P}_{1 / 2}\right)(b b)$ dissociation limit has been performed. For this purpose, the $0_{u}^{-}(b b)$ state has been described using analysis of rotationally resolved excitation spectra of luminescence from the $\mathrm{g} 0_{g}^{-}$state populated in a three-step three-color perturbation facilitated excitation scheme via the $0_{u}^{-}$state. Energies of 41 rovibrational levels, molecular constants, and potential energy curve have been determined. Energy gaps between closest rovibrational levels of the $0_{u}^{-}$and $0_{g}^{+}, 1_{u}(b b)$ states are found to be large, $\sim 6 \mathrm{~cm}^{-1}$. However, interaction of all three $0_{g}^{+}$, $1_{u}$, and $0_{u}^{-}(b b)$ states has been observed. It has been found that the $0_{u}^{-}$and $1_{u}$ electronic states are mixed by heterogeneous interactions, while their mixing with the $0_{g}^{+}$one is due to hyperfine interactions predominantly. Admixture coefficients and electronic matrix elements of the coupling between the $0_{g}^{+} \sim 1_{u}, 0_{g}^{+} \sim 0_{u}^{-}$, and $0_{u}^{-} \sim 1_{u}$ states have been estimated. Published by AIP Publishing. [http://dx.doi.org/10.1063/1.4948630]
\end{abstract}

\section{INTRODUCTION}

Perturbations in electronic states of diatomic molecules are the subject of numerous studies for several decades (see, e.g., Refs. 1-4 and references therein). One of the practical applications of the perturbations between states is perturbation facilitated optical-optical double resonance spectroscopy ${ }^{5-9}$ in which mixed intermediate states are used as gates to excited states of various symmetry. This method allows to investigate "dark" excited states that are inaccessible in direct excitation from a ground state or in a commonly used optical-optical double resonance scheme.

Some scientific groups utilized mixed valence states of iodine molecule as an intermediate ones to populate ion-pair (IP) states of the tiers, correlating with four dissociation limits of atomic ions, $\mathrm{I}^{-}\left({ }^{1} \mathrm{~S}\right)+\mathrm{I}^{+}\left({ }^{3} \mathrm{P},{ }^{1} \mathrm{D},{ }^{1} \mathrm{~S}\right)$. These states draw sufficient attention of researchers because they provide a very convenient system for understanding collisional nonadiabatic energy transfer processes, ${ }^{10,11}$ molecular perturbations, ${ }^{6,7,12,13}$ and development of theoretical models. ${ }^{14,15}$

A hyperfine interaction of the iodine molecule $B 0_{u}^{+}$and $c 1_{g}$ states, correlating with the second $\mathrm{I}\left({ }^{2} P_{3 / 2}\right)+\mathrm{I}\left({ }^{2} P_{1 / 2}\right)(a b)$ dissociation limit, was used for the $\gamma 1_{u},{ }^{5} H 1_{u},{ }^{6}$ and $\mathrm{F} 0_{u}^{+8}$ IP state optical population. The $B 0_{u}^{+} \sim 0_{g}^{-}(a b)$ hyperfine coupling allowed to describe the $h 0_{\mathrm{u}}^{-}\left({ }^{3} \mathrm{P}_{1}\right)$ ion-pair state, ${ }^{6}$ and the $g 0_{g}^{-}\left({ }^{3} \mathrm{P}_{1}\right)$ state was excited via the $B 0_{u}^{+} \sim(3) 0_{u}^{-}$coupled state. $^{9}$

Theoretical description of the hyperfine interaction of the $B 0_{u}^{+}$state with different valence states was given by Vigue et al. ${ }^{16}$ and Pique et al. ${ }^{1}$ Vigue et al. estimated the electronic

\footnotetext{
a) Author to whom correspondence should be addressed. Electronic mail: s.lukashov@spbu.ru
}

matrix elements of two major terms of hyperfine Hamiltonian corresponding to magnetic dipole and electric quadrupole interactions as $\sim 0.3 \mathrm{~cm}^{-1}$ and $0.1 \mathrm{~cm}^{-1}$, respectively. ${ }^{16}$ Similar results were reported by Pique et al. ${ }^{1}$ Experimental studies gave the coupling matrix element value of about $0.01 \mathrm{~cm}^{-1}$ similar to the theoretical estimations taking into account the Frank-Condon factor $(F C F)$ of about $0.1 .^{7,8,17-20}$

Recently, we have realized an effective population of IP states using the three-step three-color excitation scheme via the $B 0_{u}^{+}$and $0_{g}^{+}$states, correlating with the second $(a b)$ and the third, $\mathrm{I}\left({ }^{2} P_{1 / 2}\right)+\mathrm{I}\left({ }^{2} P_{1 / 2}\right)(b b)$, dissociation limits, respectively. We have shown that the perturbation of the $0_{g}^{+}(b b)$ and other two, $1_{u}$ and $0_{u}^{-}$, (bb) states allows to populate both gerade and ungerade IP state ${ }^{21-23}$

$I P \stackrel{h v_{2}}{\longleftarrow} \quad 0_{g}^{+}, 0_{u}^{-}, 1_{u}(b b) \stackrel{h v_{f}^{i}}{\longleftarrow} \quad B, v_{B}, J_{B} \stackrel{h v_{1}}{\longleftarrow} X, v_{X}, J_{X}$.

All three $(b b)$ states have been observed in condensedphase emission studies ${ }^{24}$ for the first time. In gas phase, Exton and Balla ${ }^{25}$ observed very weak luminescence near $750 \mathrm{~nm}$, following the $193 \mathrm{~nm}$ ArF laser excitation of the $D 0_{u}^{+} \mathrm{IP}$ state, which can be assigned apparently to the $D 0_{u}^{+} \rightarrow 0_{g}^{+}(b b)$ transition. Ridley et al. ${ }^{5}$ described the $0_{g}^{+}$and $1_{u}(b b)$ states in analysis of the vibrationally resolved IP $\rightarrow(b b)$ luminescence spectra.

In our previous work, ${ }^{26}$ we observed population of $\beta 1_{g}$ and $D 0_{u}^{+}$IP states from the $1_{u}$ and $0_{g}^{+}(b b)$ intermediate states, respectively, in a three-step excitation scheme in the $\mathrm{I}_{2}+\mathrm{Rg}, \mathrm{Rg}=\mathrm{He}, \mathrm{Ar}, \mathrm{Kr}, \mathrm{Xe}$ mixture. We have observed luminescence spectra and energy loss approximately $400 \mathrm{~cm}^{-1}$ in the excitation process. It has been proposed that there is optical excitation of $\mathrm{RgI}_{2}$ van der Waals complexes with the 